\title{
Using knowledge graphs and behaviour trees for feedback-aware presentation agents
}

\author{
Nils Axelsson \\ nilsaxe@kth.se \\ KTH Royal Institute of Technology \\ Stockholm, Sweden
}

\author{
Gabriel Skantze \\ skantze@kth.se \\ KTH Royal Institute of Technology \\ Stockholm, Sweden
}

\begin{abstract}
In this paper, we address the problem of how an interactive agent (such as a robot) can present information to an audience and adapt the presentation according to the feedback it receives. We extend a previous behaviour tree-based model to generate the presentation from a knowledge graph (Wikidata), which allows the agent to handle feedback incrementally, and adapt accordingly. Our main contribution is using this knowledge graph not just for generating the system's dialogue, but also as the structure through which shortterm user modelling happens. In an experiment using simulated users and third-party observers, we show that referring expressions generated by the system are rated more highly when they adapt to the type of feedback given by the user, and when they are based on previously grounded information as opposed to new information.
\end{abstract}

\section{CCS CONCEPTS}

- Human-centered computing $\rightarrow$ User models; • Software and its engineering $\rightarrow$ Entity relationship modeling; • Computing methodologies $\rightarrow$ Discourse, dialogue and pragmatics.

\section{KEYWORDS}

Wikidata, dialogue, dialog, system, grounding, feedback, knowledge, relation, graph, behaviour tree, behavior tree

\section{ACM Reference Format:}

Nils Axelsson and Gabriel Skantze. 2020. Using knowledge graphs and behaviour trees for feedback-aware presentation agents. In IVA '20: Proceedings of the 20th ACM International Conference on Intelligent Virtual Agents (IVA '20), October 19-23, 2020, Virtual Event, Scotland UK. ACM, New York, NY, USA, 8 pages. https://doi.org/10.1145/3383652.3423884

\section{INTRODUCTION}

In dialogue, participants adapt to each other with the help of feedback. Feedback allows a listener to tell the speaker that information is grounded or not grounded on multiple levels. As part of a larger effort to model this process for social robots, we are investigating how a presenting agent can adapt an art presentation to how its audience reacts. In this art presentation scenario, which is intentionally more one-sided than an open conversation between two humans, the audience mostly reacts with short positive or negative

\section{c) (i) $\ominus$}

This work is licensed under a Creative Commons Attribution-NonCommercialNoDerivs International 4.0 License.

IVA '20, October 19-23, 2020, Virtual Event, Scotland UK

(C) 2020 Copyright held by the owner/author(s).

ACM ISBN 978-1-4503-7586-3/20/09.

https://doi.org/10.1145/3383652.3423884 feedback. It is the job of the agent to use this limited information to make its presentation as appropriate as possible. Figure 1 shows an example dialogue that can be generated by the system we propose in this paper.

\begin{tabular}{lllr}
\hline Speaker & Speech & Interpretation / action & GC \\
\hline Robot: & Da Vinci was b- & & Att \\
User: & Who? & Negative und. & Und \\
Robot: & Leonardo da Vinci... & Alternative reference & Und \\
User: & Who was that? & Negative und. & Und \\
Robot: & the artist... & & Und \\
User: & Ah, OK. & Positive und. & Und \\
Robot: & was born in 1452. & & Acc \\
User: & $\ldots$ & Not meeting GC & Acc \\
Robot: & $\ldots$ around 600 years & & \\
& ago. & Alternative reference & $A c c$ \\
User: & $\ldots$ & Not meeting GC & $A c c$ \\
Robot: & Right? & Eliciting acceptance & Acc \\
User: & Yeah, sure, I got it. & Positive acceptance & $A c c$
\end{tabular}

Figure 1: An example of the output of the system operating as described in Section 3. GC is grounding criterion, att is attention, und is understanding and acc is acceptance; see Section 2.1.

In a previous paper [5], we have shown how behaviour trees can be used to model the grounding process and the interaction between a robot presenting a piece of art to its audience. We used the art presentation scenario in an experiment where users showed a preference towards a mode that adapted to their feedback over one that used a pre-determined script [5]. One limitation of our previous model was the coarseness of its grounding model, and how it could only present and ground one pre-written utterance at a time.

This paper has three contributions. First, we extend our previous behaviour tree model to support incremental generation of utterances, broken down into what is being spoken about (theme) and what is being said about the theme (rheme). This extension allows the system to interpret user feedback by taking into account what utterance part they are reacting to. Second, we show how such incremental presentations can be generated from a knowledge graph, and how the graph can be used to keep track of the grounding status of the different entities and relations in the presentation. Third, we present an evaluation showing that subjects prefer a system that is sensitive to different forms of user feedback, and that can use the grounding status of previous information to adapt the presentation. 


\section{BACKGROUND}

\subsection{Grounding and feedback}

Clark [18] defines grounding as establishing information as part of common ground well enough for the purposes of the discourse, where common ground is the collective knowledge and beliefs of participants in a conversation. The grounding criterion of an exchange is the level at which the speaker and listener collectively believe feedback must be given to ground the thing being talked about [19]. Clark [18] describes grounding in terms of four levels attention, hearing, understanding and acceptance. Allwood et al. [3] make a similar division between contact, perception, understanding and agreement. Attention or contact is if the message put out by the speaker is arriving at the listener. Hearing or perception is identifying the message put out by the robot, and understanding refers to the listener being able to derive the intention of the message. Acceptance or agreement is what attitude the listener has towards the understood message; if they accept or reject it.

Upward completion is a process where negative feedback on a low level implies negative feedback on all higher levels; negative hearing must imply negative understanding and acceptance, since one cannot understand or accept what one has not heard. Conversely, downward evidence refers to positive feedback on a high level implying positive feedback on all lower levels; if one accepts a statement, then one must also have understood, heard and paid attention to it [18].

Allwood et al. [3] define feedback as one of the interactive functions "whereby speakers manage the flow of interaction". It is a general name for all reactions that allow a listener to communicate positive or negative grounding on one of the levels defined above [2]. Yngve [51] defines backchannels as those reactions of this type that are not intended to take the turn from the speaker.

\subsection{Knowledge graphs}

Knowledge graphs were introduced by Minsky [35], who called them semantic networks for use in question-answering systems. Minsky defines a semantic network as a graph of nodes and edges, where nodes are concepts or entities, and the edges between the nodes represent a conceptual or practical relationship of some type between the two nodes [35, p. 153]. More recently, projects like DBPedia [4] and Freebase [11] were started to extract information in this format from the generally unstructured text of Wikipedia. The format of the data is typically referred to as an RDF triple; the source of an edge is the subject, the label of the edge is the predicate or property, and the other end of the edge is the object [21].

Wikidata is a relational database launched in 2012 by the Wikimedia foundation [48]. It is a successor to Freebase, and originally contained much of the same data [37]. Wikidata serves as an underlying database for multiple Wikimedia projects, linking articles and entries about the same entities across wikis. It is thus not only an interpretation of data that is already in Wikipedia, but also an underlying engine supplying various Wikimedia projects with structured data. The database can be accessed freely for non-commercial or commercial purposes through a public API [23].

Wikidata calls the nodes of its graph items and the edges between nodes properties. The pair of a property and the item or constant on the other end is called a statement about the subject. All items are assigned a unique, arbitrary identifier starting with $Q$, while types of properties start with $P$. For example, Leonardo da Vinci has the identifier Q762. Some properties are specific to certain types of items, and others are more common. For example, Da Vinci has the common instance of (P31) property to human (Q5). A selection of properties connected to Leonardo da Vinci can be seen in Figure 4. Properties can either target items or constants. A constant is some value that does not get its own item ID, but is contained in the statement. Dates are typically constants.

Wikidata and DBPedia have been used to fill in templates for natural language generation in spoken dialogue systems [26, 33]. Both databases have been used to generate text from triples [17, 24, 25]. Datasets like the WebNLG 2017 dataset are typically used to train neural models that can generate English text based on triples [30, 36, 38]. Our approach is not data-driven in this way, but specifically the work of Elder et al. [22], who neurally extract an intermediate knowledge graph representation from triples, holds interest for future work.

\subsection{Feedback-aware dialogue systems}

The idea of creating a system that has an internal estimation of the user's mental state is known as user modelling. Rich [40] adressed the issue of short-term adaptation in the Grundy bookrecommending system by applying stereotypes to the information known about the user. Kobsa [32, 49] explored the advantages and dangers of user-modelling, but mostly from a perspective of simple question-answering systems, tax form assistance, or programming optimisation.

Several studies have shown that educational systems that provide feedback to users are more efficient teachers compared to systems that do not, or to those that use random behaviours [16, 46, 47]. While many studies have addressed the problem of how dialogue systems can give feedback to users on the different grounding levels discussed in 2.1 [ $31,34,42,43$ ], there are relatively few studies of how user feedback can be modelled in dialogue systems. Recently, Buschmeier and Kopp [13, 15] have presented models for how dialogue systems can map user reactions to internal approximations of their user state using Bayesian models. At each time step, feedback from the user is combined with the system's previous approximation of the user's grounding state to form the new approximation of the user's grounding state. The user's feedback affects the system's attributed listener state $(A L S)$ through various features, like at what grounding level the feedback or backchannel is classified, and the timing of how it was delivered in the context of the discourse [14]. Other studies have investigated how to interpret user feedback (including multi-modal aspects such as prosody and gaze) $[8,9,41,45]$.

In this paper, we build on the ideas by Buschmeier and Kopp. In Section 3.3, we suggest how a presenting system can take the attributed listener state and use this classification of the user's behaviour for deciding what and how to present next.

\subsection{Information structure}

In a sentence, the theme is what something is being said about, and the rheme is what is being said about the theme [29,39]. These 
terms imply that the entity referred to in the theme is something previously grounded, while the rheme is new information. A knowledge graph triple (see Section 2.2) can be seen as a theme-rheme pair; the subject is the theme, and the predicate and object make up the rheme [27, 50].

Other authors [28] have used the terms topic and comment for the same concepts as theme and rheme respectively, although it is not universal that they are given the same definitions. Brown and Yule [12] define theme as the leftmost constituent in a sentence and the topic as the thing being spoken about. In the dialogue synthesis examples we present in this paper, the topic entity is always also the leftmost entity in the synthesised sentence; therefore, we have no need for distinction, and simply call them theme and rheme.

\subsection{Behaviour trees}

A behaviour tree is a model of a control system where independent tasks are modelled as sub-trees between which the system can switch [20]. Nodes return only the three values: SUCCESS, FAILURE or RUNNING to their parents. Nodes may have several ordered children; sequence and selector nodes are such multi-child nodes that run a sub-set of their children depending on the return values of the children that are executed.

Selector Runs its children until one returns SUCCESS or RUNNING, at which time the selector also returns that value. If all children return FAILURE, the selector also returns FAILURE. Denoted with a ? symbol in Figures 2 and 3.

Sequence Runs its children until one returns FAILURE or RUNNING, at which time the selector also returns that value. If all children return SUCCESS, the selector also returns SUCCESS. Denoted with a $\longrightarrow$ symbol in Figures 2 and 3 .

The nodes in the tree are processed (ticked) from left to right (starting at the top) at a certain rate. The tree we present in this paper runs at $10 \mathrm{~Hz}$. At each tick, the tree will return one of the three values mentioned above. Thus, a sequence that succeeds at one tick might fail at the next tick (which might be in the middle of an utterance), rendering a different behaviour. We believe that this real-time nature of behaviour trees makes them appropriate for incremental processing of feedback and incremental production of speech reacting to that feedback, in the line of $[6,44]$. Additionally, the independence of sub-trees means that the speech synthesis component does not need to know the exact implementation of the grounding component.

Tree models in general have long been used to model presenting and conversing systems $[7,10]$, but behaviour trees appear to have been limited to video games and non-social robotics until recently.

\section{PROPOSED INTERACTION MODEL}

Our primary contribution in this paper is the combination of a behaviour tree with a grounding component that is directly connected to an underlying knowledge graph (generated from Wikidata). For each user in the interaction, individual knowledge graph properties are marked as either grounded or un-grounded, depending on the feedback that is received. The behaviour tree presented here is an extension of the one presented in a previous paper [5]. The extensions to the system consist of both changes to the tree and to the external components used to synchronise the tree's operation. They are described separately below.

The interaction model was implemented in Java and Kotlin as a module for the FurhatOS platform, using the Furhat [1] robot head. For speech synthesis, Amazon Polly was used.

\subsection{Overview of interaction behaviour tree}

The extended behaviour tree is illustrated in Figure 2. The coloured nodes labelled "ensure user attention", "ensure positive hearing", "ensure positive understanding" and "ensure positive acceptance/interest" are collapsed to make the figure smaller. The implementation of the attention grounding subtree ("ensure user attention") is seen in Figure 3; the others are equivalent for their respective grounding level.

The tree in Figure 2 should be read from left to right and top to bottom. Thus, its subtrees do the following behaviours, in order. As its first priority, the tree will invite users to its presentation if there are no users already invited. If this succeeds, the tree moves on to interacting with its newly or previously invited users.

The children of "interact with user", a selector, are set up in such a way that "robot's initiative" never executes if the user has the turn. The user has the turn if the "user's initiative" subtree returns SUCCESS. This happens if the user speaks and is allowed the turn by the system. If it is the user's turn, the system looks at the user and gives them evidence that it is listening. Any speech is logged and timestamped for processing when the robot re-gains the turn.

If the user does not have the turn, the robot takes or already has the turn. It then executes its presentation, after handling any speech and backchannels that have happened since the last time this subtree ran. This happens in the "react to user input" subtree. Speech and backchannels can have the effect of un-grounding or grounding certain information in the knowledge graph, which also serves as a user model (see Section 3.3).

According to the principles of downward evidence and upward completion (as described in section 2.1), the system grounds attention, hearing, understanding, and acceptance in order; how this is done in relation to the incremental utterances based on the knowledge graph is presented in Section 3.3.

Finally, the system speaks, by first validating that no previous part of the current utterance has been retroactively un-grounded by user feedback, and then speaking the current utterance, returning RUNNING if speech synthesis is not done. This can fail if the system runs out of ways to refer to an item; if this happens, the system falls back on trying another property to present in its "try other line" leaf. If the system cannot find a new such property, which could be because there is nothing more to present, or because the system has run out of time or does not want to interact with the user anymore, the presentation ends.

\subsection{Utterance generation from knowledge graph}

We generate a local knowledge graph from Wikidata queries. Only properties the system can present are part of the compiled graph; this is a pre-written list of properties we have manually paired with strings. In our generated graph, items can be marked as implicitly part of common ground because of the situation; for example, in a 


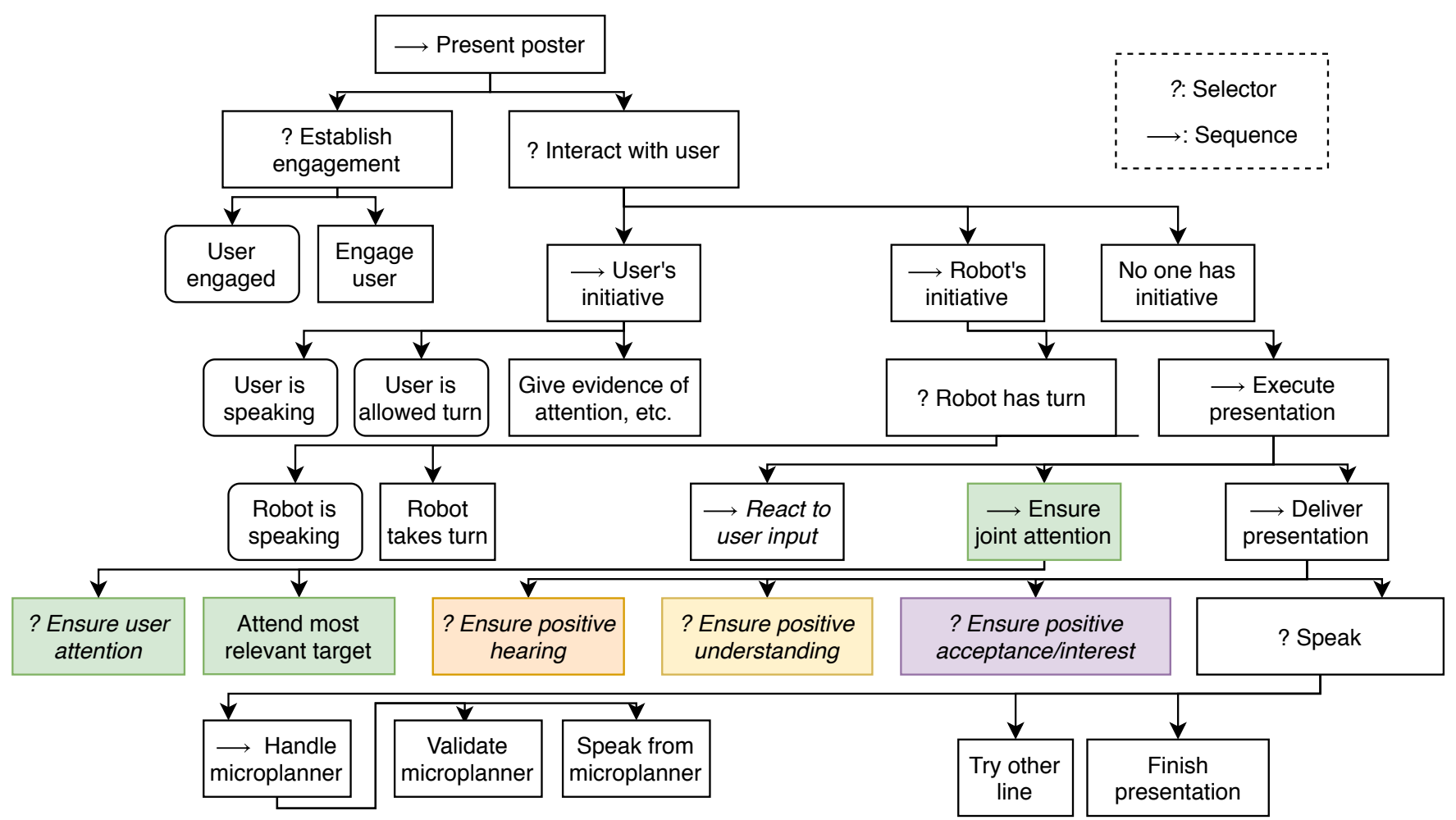

Figure 2: The extended behaviour tree. The implementations of the four coloured nodes with italic text at the bottom of the tree are omitted to save space. The implementation of "ensure user attention" (light green) is found in Figure 3; the other coloured omitted grounding subtrees are equivalent for their respective grounding level.

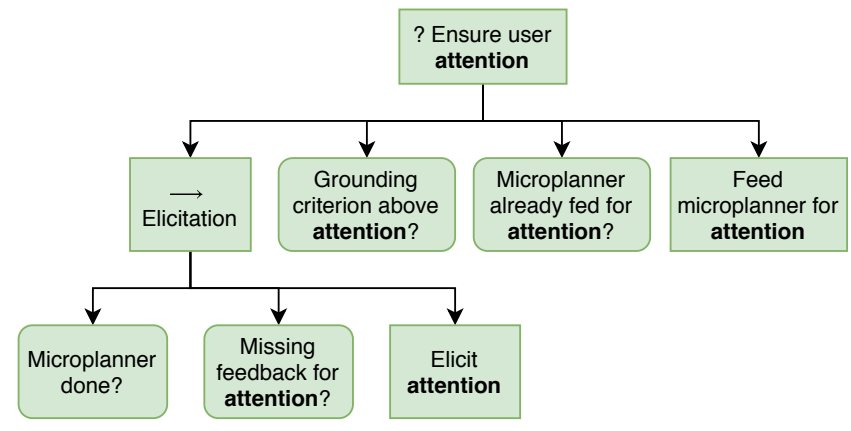

Figure 3: How the system grounds attention, an elaboration of parts of the tree not seen in Figure 2 .

room with a painting on the wall, the painter and painting, as well as the P170 ("creator") property between them, are marked as part of common ground before the interaction even starts. It is implicit that there is a painting, a painter and a creator-created relationship between them; the unknown part to the audience is the identities of the painter and painting, which the agent can present.

A Wikidata constant (see Section 2.2) cannot be the subject of a statement. In our interpreted graph, constants are represented by nodes like any item; we therefore do not have this limitation. We insert constants referring to deictic information like the current year and pronouns. To allow the system to compare different values of constants of the same type, we also insert properties comparing constants of the same type to each other. For example, year constants can be compared to all other year constants. Since this would result in an unreasonable number of properties scaling quadratically with the number of year constants, we create these properties dynamically only between grounded year constants during the reference synthesis process. This insertion of properties allows the system to refer to the year 1452 as " 568 years ago", and to ground future rhemes in previously introduced information "Vincent van Gogh died in 1890, five years after his father died." The same comparison process could be extended to other types of easily comparable constants, but the comparison needs to be manually implemented for each type of Wikidata constant.

The system keeps track of what property or properties it is currently presenting through an agenda component, which can be queried to select a new set of such properties based on the grounding state of the knowledge graph for the currently invited audience. When the "try other line" leaf queries the agenda for a new line to present, it also initialises a microplanner component with empty slots for the theme and rheme of the selected line. The theme and rheme are further broken down into sub-slots, which we call unresolved utterance parts.

An unresolved utterance part is a template for what type of information the grounding subtrees should try to generate for that part of the utterance. If the agenda chooses the Wikidata statement Q762 $\longrightarrow$ P569 $\longrightarrow$ "1452" ("Leonardo da Vinci was born in 1452") 


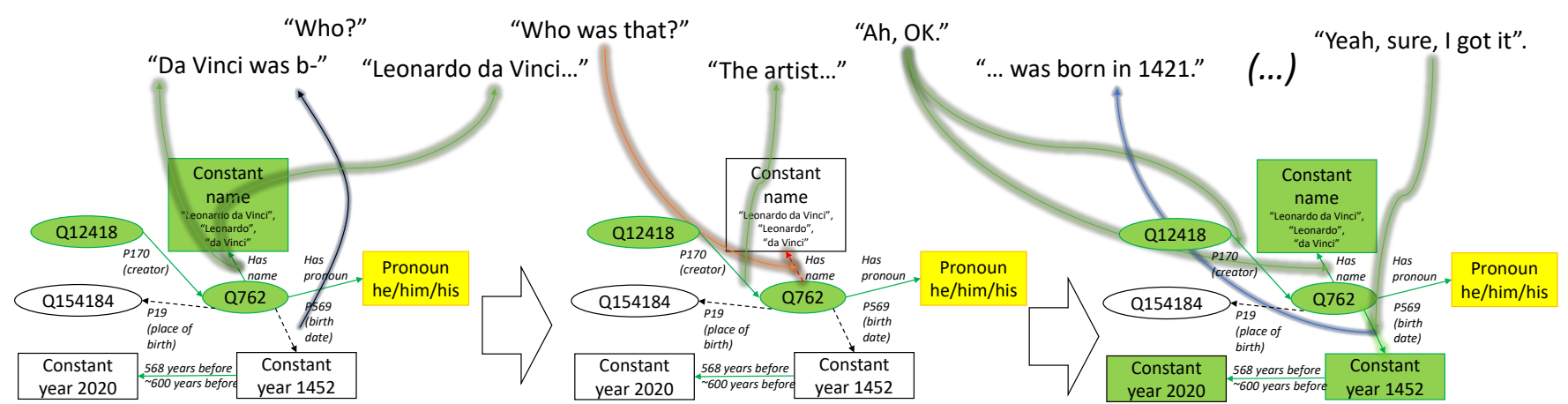

Figure 4: A visualisation of what properties are grounded on the grounding level understanding before, in the middle of, and after the conversation shown in Figure 1. Green nodes and edges represent grounded entities and properties; ungrounded edges are also dashed. The pronoun item is coloured yellow since grounding does not apply to pronouns. Constants (square boxes) are coloured in green if the system can relate them to $Q 762$.

as the next statement for the system to present, then the microplanner is initialised with three unresolved utterance parts; one for a reference to the theme item, Q762, one for the connecting string "was born", and one for the reference to a prepositional phrase referring to the year 1452. This example is illustrated in Figures 1 and 4.

For each slot in the microplanner, the system keeps track of its grounding criterion, which is the minimum level at which the user must leave positive feedback for the system to move on with the utterance. If the feedback does not meet the grounding criterion level, the system will elicit feedback from the user. By default, the system assumes a grounding criterion of attention for the theme and understanding for the rheme, but this can change as feedback is received; see Section 3.3. This happens when the user reacts with negative understanding in Figure 1.

The grounding subtrees illustrated in Figure 3 iterate over the microplanner and check if there are any unresolved utterance parts whose grounding criterion are at or below the grounding level of the tree. If there are any such unresolved utterance parts, and they have not already been paired with a resolved utterance part by the subtree, then the subtree generates such a resolved utterance part.

For connecting strings like "was born" in Figure 1, the resolved utterance part always contains the same static string for a given property; we pre-wrote these for each property our system can present. An example is "was born" for P569. We will leave the adaptation of verb phrases for future work.

If the unresolved utterance part represents a reference to an item, which can happen both as part of the theme and the rheme, then the grounding sub-tree creates a reference by looking at grounded properties and entities that are part of common ground for the current set of users. Every reference to the item is generated through a recursive algorithm that traverses every grounded edge, or grounded property, of the graph at most once. Edges in the graph are optionally paired with text that can be used to refer to the target or source of the edge in isolation, or text that can be circumfixed around another reference to relate it to the item.

For the property P569, "birth date", the strings are "the birth date" when traversed forwards in isolation, "the birth date of..." as a prefix when traversed backwards, and ", who was born..." as a prefix when traversed forwards attributively. These allow the system to refer to the birth date itself as "the birth date" or "the birth date of (reference to the subject of the statement)", and to the entity on the other end of the birth date statement as "(reference to subject), who was born (prepositional phrase of object)". The list of every possible reference is sorted to de-prioritise repeated references and prioritise references that have succeeded in the past. The best reference by these measures is given to the microplanner as a resolved utterance part.

A resolved utterance part not only contains text that the system should speak, but also prosodic information, for example if the utterance part should be slowed down. For example, the hearing subtree can determine that it is the second time it is trying to refer to an entity, and intentionally re-use a previous reference, but louder and slowed down as an adaptation strategy.

\subsection{Feedback and adaptation strategies}

The list of resolved utterance parts is passed to speech synthesis through the "speak from microplanner" leaf at the bottom of Figure 2. If the user responds with feedback, either by taking the turn or by giving a backchannel in the middle of the utterance, the react to user input subtree routes the feedback to the knowledge graph, as shown in Figure 4.

The most recently finished utterance part at the time the user's speech started is selected as the feedback target. This process assumes that the feedback has been classified as positive or negative attention, hearing, understanding or acceptance. Feedback towards a reference routes the feedback to the item referred to by the reference, increasing the user's familiarity with the item if the feedback is positive, or reducing the familiarity if the feedback is negative. The familiarity value is later used to decide if more or less detailed readings of constants or properties should be used ("da Vinci" instead of "Leonardo da Vinci" in Figure 1). Positive feedback below the grounding criterion is not routed.

Feedback is routed from the resolved utterance part to the parts of the knowledge graph that were used to generate the utterance part. An example of this is when negative feedback is routed from a reference to every property that was crossed to generate the 
reference. This can be seen in the middle of Figure 4; the user says "Who was that?", ungrounding the statement that Q762 has the name "Leonardo da Vinci". The statement is re-grounded one turn later as the user displays positive feedback.

When positive feedback is received for the final rheme of a line, the feedback grounds the properties that the line expressed. The grounding happens on the level of the received feedback, with downward evidence. In Figure 1, the final received feedback is positive acceptance; thus, the statement Q762 $\longrightarrow$ P569 $\longrightarrow$ "1452" is grounded on the level of acceptance, and downward evidence causes it to also be grounded on every level below that, as seen on the right of Figure 4. The system can now use this property to generate additional references to Q762 ("Leonardo da Vinci, who was born in 1452..."), as well as the constant of 1452 ("the birth year of Leonardo da Vinci") and 2020 ("around 600 years after the birth year of Leonardo da Vinci").

Each of the grounding subtrees in Figures 2 and 3 has a different strategy to adapt to negative feedback on its grounding level. The attention subtree does not change its references, and will elicit attention upon negative attention. The hearing subtree will repeat its first reference with gradual slowdown and raised volume, and try to replace shortened readings of constants with longer readings. The understanding subtree finds alternative readings by traversing different properties to generate new references. The acceptance subtree marks the properties towards which negative acceptance was received as disliked by the user, making them less likely to appear in the future, and then intentionally does not generate a reference, making the tree choose a new line and come back to the failed line in the future, when there may be more ways to express the failed property. These different adaptation strategies are achieved by having different implementations of the leaves seen in Figure 3; the structure of the trees is still equivalent.

\section{EVALUATION}

An experiment was set up to evaluate the system's ability to generate valid presentations from randomly chosen paintings from the Louvre in Wikidata, and adapt the presentation according to feedback from a user. To be able to systematically contrast different forms of adaptation, test participants (crowd workers) were asked to rate alternative system responses given a preceding dialogue context. The dialogues to be rated were generated by letting a simulated user interact with the system and recording the exchange. The simulated user was represented with pre-recorded speech in the dialogues; a large bank of sound clips for negative and positive feedback was recorded and picked from randomly to reduce repetition between dialogues. The simulated user was set up so that all feedback it gave would be correctly classified by the system.

With this approach, we sought to answer two questions: (1) whether referring expressions that are based on previously grounded information are preferred to those that are not, and (2) if different adaptation strategies are evaluated as better or worse depending on whether the user's feedback is negative hearing or negative understanding.

Two scenarios were created, and were intentionally kept similar and comparable in order to allow the options given to the experiment participants to be equivalent for each recording. The two scenarios are elaborated upon in Section 4.1. The 120 generated dialogues were split evenly across scenarios and feedback type. Each dialogue was evaluated by five separate participants, giving 300 rankings for negative hearing and 300 rankings for negative understanding. Negative attention and acceptance were omitted. Negative attention proved hard to represent in audio form. Negative acceptance demanded an adaptation strategy that was very different from the other forms of negative feedback, namely moving on to something else and then coming back to the statement later.

The experiment was run remotely over Amazon's Mechanical Turk platform. The ages and gender of participants were not collected. Participation was limited to Mechanical Turk's "Master Worker" category of users. 27 unique users participated in the task. Participants were paid $\$ 0.2$ for each dialogue they rated.

\subsection{Scenarios}

Each of the 120 dialogues was generated with a randomly chosen painting from the Louvre, to verify the generalisability of our model. In both scenarios, the robot would welcome the user to the presentation, and then attempt to present three lines about the chosen painting and painter. After the first line, the user would always react with weak positive understanding. After the second line, the user would react with stronger positive understanding. After the robot had said the theme of the third line, the user would interrupt with either negative hearing or understanding. The robot would attempt to repeat the theme, and the user would respond with a stronger variant of feedback at the same grounding level. At this point, evaluators were presented with four options for what the system should say next, listed below.

The two scenarios differed in what the system would use as the theme of the second line. In the first scenario, the second line was a fact about the chosen painting, and in the second scenario, the second line was a fact about the painter. The four options were always the following four, presented in a random order. The contents of the options were generated independently of the adaptation strategies mentioned in Section 3.3, but one of the options was the system's preferred adaptation depending on the user's feedback - "repeat" if negative hearing, and "alt. grounded" if negative understanding.

Repeat A repetition of the painter's name, without slow-down.

Repeat slowly A repetition of the painter's name, slowed down in speech synthesis by $25 \%$, to clarify.

Alt. grounded A rephrased reference referring to the second line of the scenario, of the type "(the painter's name), (attributive reading referring to the second line)".

Alt. ungrounded An alternative rephrased reference, referring to something similar to the second line of the scenario, of the type "(the painter's name), (attributive reading referring to the something else)", but referring to something that was never said in the dialogue. The system was only allowed to use properties it could have presented as the second line.

\subsection{Results}

The ranks given by test participants for the 60 dialogues where the user responded with negative understanding were tested for ordering with the Friedman test. The same was done for the 60 dialogues where the user responded with negative hearing. The 


\begin{tabular}{ll}
\hline Speaker & Speech \\
\hline Robot: & You are welcome to join in. The painter was called \\
& Vincent van Gogh. \\
User: & Uh-huh. \\
Robot: & He was influenced by Paul Gaugin. \\
User: & Okay. \\
Robot: & Vincent van Gogh was- \\
User: & Sorry, what? \\
Robot: & Vincent van Gogh... \\
User: & Sorry, who was that? \\
\hline Alt. & Vincent van Gogh, who was influenced by Paul Gau- \\
grounded & gin, was born in Zundert. \\
Alt. un- & Vincent van Gogh, who died in 1890, was born in \\
grounded & Zundert. \\
Repeat & Vincent van Gogh was born in Zundert. \\
Repeat & Vincent van Gogh was born in Zundert. \\
slowly &
\end{tabular}

Figure 5: An example instance of the second scenario detailed in Section 4.1. The italic text in the "repeat slowly" option denotes slowed down speech.

Friedman test reported an overall ordering effect for both hearing $(\chi(3)=131.3, p<0.001)$ and understanding $(\chi(3)=317.09, p<$ $0.001)$. Post-hoc tests were performed to determine the significant orderings in the ranks. The orderings are presented in Table 1. There was no significant difference between a reference to grounded information and a repetition without slowdown in the case where the user gave negative hearing; all other orderings were significant $(\mathrm{p}<0.001)$.

\begin{tabular}{llcc}
\hline Scenario & Option & Mean rank & Sign. comp.? \\
\hline \multirow{4}{*}{ Hearing } & Repeat slowly & 2.19 & \}$* * * *$ \\
& Alt. grounded & 2.27 & \} ns \\
& Repeat & 2.30 & \}$* * * *$ \\
& Alt. ungrounded & 3.24 & \\
\hline \multirow{4}{*}{ Understanding } & Alt. grounded & 1.58 & \multirow{2}{*}{\}$* * * *$} \\
& Repeat slowly & 2.39 & \}$* * * *$ \\
& Repeat & 2.58 & \}$* * * *$
\end{tabular}

Table 1: The mean ranks of the options for the dialogues where the simulated user gave negative hearing (top) and understanding (bottom). Options are ordered by mean rank for each scenario; 1 is the best option, and 4 the worst.

As the results show, different adaptation strategies are preferred, depending on the grounding level of the user feedback. In case of negative hearing, a slowed-down repetition is preferred, while a rephrasing using grounded information or a simple repetition are quite close in terms of ranking. In case of negative understanding, a rephrasing using grounded information is clearly preferred. In both cases, rephrasing using non-grounded information is ranked lowest. This shows that it is very important for the system to keep track of the grounding status of information in the graph, in order to be able to rephrase in an adaptive way.

\section{CONCLUSIONS AND DISCUSSION}

In this paper, we have presented a model for generating interactive presentations that take user feedback into account, allowing the agent to adapt accordingly. Knowledge graphs (from Wikidata) are used to generate these presentations incrementally, and to keep track of the grounding status of the different items and properties in the graph. An evaluation using third-party observers showed that referring expressions generated by the system are rated more highly when they adapt to the type of feedback given by a simulated user, and when they are based on previously grounded information as opposed to new information.

The various neural text generation models developed based on the WebNLG 2017 dataset (see Section 2.2) show that this is a viable and preferred way to synthesise text from knowledge graph triples. However, for grounding purposes, it is important that the system knows what it is saying, not only on a macro level, but also on a microplanner level, so that user feedback can be mapped to the smallest piece that corresponds to what they did or did not attend, hear, understand or accept. For this reason, we believe that a purely end-to-end approach is difficult to apply to a grounding-based presenting system like the one we are presenting here.

The way we generate utterances from properties only takes one property at a time into consideration. In reality, what we know about an entity constrains how we can refer to that thing, but we consider all those constraints at the same time [12]. The system described in Section 3.3 is incapable of creating references like "the French guy", because it makes use of both the fact that the entity was French and that the entity was a man. Two references in a row connected by commas almost allow the system to say equivalent phrases ("the man, who was French, ..."), but this is not a full solution. A constraint-based system would also be able to implement constraints like "that painter" referring only to one specific painter (the most recently referred painter), while the reference "the painter" could be ambiguous. We leave this for future work; our grounding implementation would still be a sound base for such an extension and is our main contribution.

The approach we presented in Section 3.3 allows a system to ground and compare new information with previously presented information. However, the system currently does not have a good way to evaluate which comparisons and references are interesting to present for the user. We currently use a heuristic based on how many properties the reference generation algorithm has to cross to arrive at a reference, but some good, contextual references may have to cross many properties ("Leonardo da Vinci was born in the same year that the Gutenberg Bible was printed"). This is where the advantages of a data-driven approach become clear. If the dialogue system is trained on text and triples extracted from human-written sources, then it could learn to express statements that humans think are interesting to express. We leave this aspect for future work.

Although the system is fully implemented and can be run with real users, we still need more sophisticated methods for classifying user feedback as negative or positive on the different grounding levels, if users are to interact with the system in a natural way. The Bayesian approach to feedback classification proposed by Buschmeier and Kopp [14] could be interesting for this purpose. We are currently addressing this problem using Wizard-of-Oz data. 


\section{ACKNOWLEDGEMENTS}

This work is supported by the SSF (Swedish Foundation for Strategic Research) project COIN. We would also like to thank the anonymous reviewers for their helpful comments.

\section{REFERENCES}

[1] Samer Al Moubayed, Jonas Beskow, Gabriel Skantze, and Björn Granström. 2012 Furhat: a back-projected human-like robot head for multiparty human-machine interaction. In Cognitive behavioural systems. Springer, 114-130.

[2] Jens Allwood. 1988. Om det svenska systemet för språklig återkoppling. Svenskans beskrivning 16, 1 (1988), 89-106.

[3] Jens Allwood, Joakim Nivre, and Elisabeth Ahlsén. 1992. On the semantics and pragmatics of linguistic feedback. Journal of semantics 9, 1 (1992), 1-26.

[4] Sören Auer, Christian Bizer, Georgi Kobilarov, Jens Lehmann, Richard Cyganiak, and Zachary Ives. 2007. DBPedia: A nucleus for a web of open data. In The semantic web. Springer, 722-735.

[5] Nils Axelsson and Gabriel Skantze. 2019. Modelling Adaptive Presentations in Human-Robot Interaction using Behaviour Trees. In Proceedings of the 20th Annual SIGdial Meeting on Discourse and Dialogue. 345-352.

[6] Timo Baumann and David Schlangen. 2012. INPRO_iSS: A component for justin-time incremental speech synthesis. In Proceedings of the ACL 2012 System Demonstrations. 103-108.

[7] Alan W Biermann, Curry I Guinn, D Richard Hipp, and Ronnie W Smith. 1993 Efficient collaborative discourse: A theory and its implementation. In HUMAN LANGUAGE TECHNOLOGY: Proceedings of a Workshop Held at Plainsboro, New Jersey, March 21-24, 1993.

[8] Dan Bohus and Eric Horvitz. 2014. Managing human-robot engagement with forecasts and... um... hesitations. In Proceedings of the 16th international conference on multimodal interaction. 2-9.

[9] Dan Bohus and Eric Horvitz. 2016. Models for multiparty engagement in openworld dialog. (2016).

[10] Dan Bohus and Alexander I Rudnicky. 2009. The RavenClaw dialog management framework: Architecture and systems. Computer Speech \& Language 23, 3 (2009), 332-361.

[11] Kurt Bollacker, Colin Evans, Praveen Paritosh, Tim Sturge, and Jamie Taylor 2008. Freebase: a collaboratively created graph database for structuring human knowledge. In Proceedings of the 2008 ACM SIGMOD international conference on Management of data. 1247-1250.

[12] Gillian Brown and George Yule. 1983. Discourse analysis. Cambridge university press.

[13] Hendrik Buschmeier and Stefan Kopp. 2011. Towards conversational agents that attend to and adapt to communicative user feedback. In International Workshop on Intelligent Virtual Agents. Springer, 169-182.

[14] Hendrik Buschmeier and Stefan Kopp. 2012. Using a Bayesian model of the listener to unveil the dialogue information state. In SemDial 2012: Proceedings of the 16th Workshop on the Semantics and Pragmatics of Dialogue.

[15] Hendrik Buschmeier and Stefan Kopp. 2014. A dynamic minimal model of the listener for feedback-based dialogue coordination. In DialWatt-SemDial 2014: Proceedings of the 18th Workshop on the Semantics and Pragmatics of Dialogue.

[16] Huili Chen, Hae Won Park, and Cynthia Breazeal. 2020. Teaching and learning with children: Impact of reciprocal peer learning with a social robot on children's learning and emotive engagement. Computers \& Education 150 (2020), 103836.

[17] Andrew Chisholm, Ben Hachey, and Will Radford. 2017. Learning to generate onesentence biographies from Wikidata. Association for Computational Linguistics (ACL).

[18] Herbert H Clark. 1996. Using language. Cambridge university press.

[19] Herbert H Clark and Edward F Schaefer. 1987. Collaborating on contributions to conversations. Language and cognitive processes 2, 1 (1987), 19-41.

[20] Michele Colledanchise and Petter Ögren. 2018. Behavior trees in robotics and AI: An introduction. CRC Press.

[21] World Wide Web Consortium et al. 2014. RDF 1.1 concepts and abstract syntax. (2014).

[22] Henry Elder, Jennifer Foster, James Barry, and Alexander O'Connor. 2019. Designing a Symbolic Intermediate Representation for Neural Surface Realization. In Proceedings of the Workshop on Methods for Optimizing and Evaluating Neural Language Generation. 65-73.

[23] Wikimedia foundation and anonymous editors. accessed July 17th, 2020. Wikidata: Introduction - Wikidata. https://www.wikidata.org/wiki/Wikidata: Introduction.

[24] Claire Gardent, Anastasia Shimorina, Shashi Narayan, and Laura PerezBeltrachini. 2017. Creating Training Corpora for NLG Micro-Planners. In Proceedings of the 55th Annual Meeting of the Association for Computational Linguistics (Volume 1: Long Papers). Association for Computational Linguistics, Vancouver Canada, 179-188. https://doi.org/10.18653/v1/P17-1017
[25] Claire Gardent, Anastasia Shimorina, Shashi Narayan, and Laura PerezBeltrachini. 2017. The WebNLG challenge: Generating text from RDF data. In Proceedings of the 10th International Conference on Natural Language Generation. 124-133.

[26] Lorenzo Gatti, Chris van der Lee, and Mariët Theune. 2018. Template-based multilingual football reports generation using Wikidata as a knowledge base. In Proceedings of the 11th International Conference on Natural Language Generation. 183-188.

[27] Claudio Gnoli. 2018. Classifying phenomena, part 4: Themes and rhemes. KO KNOWLEDGE ORGANIZATION 45, 1 (2018), 43-53.

[28] Jeanette K Gundel. 1988. Universals of topic-comment structure. Studies in syntactic typology 17, 1 (1988), 209-239.

[29] Michael AK Halliday. 1967. Notes on transitivity and theme in English: Part 2. fournal of linguistics 3, 2 (1967), 199-244.

[30] Glorianna Jagfeld, Sabrina Jenne, and Ngoc Thang Vu. 2018. Sequence-toSequence Models for Data-to-Text Natural Language Generation: Word-vs. Character-based Processing and Output Diversity. In Proceedings of the 11th International Conference on Natural Language Generation. 221-232.

[31] Frédéric Kaplan and Verena Hafner. 2004. The challenges of joint attention. (2004).

[32] Alfred Kobsa. 1990. User modeling in dialog systems: Potentials and hazards. AI \& society 4, 3 (1990), 214-231.

[33] Huiting Liu, Tao Lin, Hanfei Sun, Weijian Lin, Chih-Wei Chang, Teng Zhong, and Alexander Rudnicky. [n.d.]. RubyStar: A Non-Task-Oriented Mixture Model Dialog System. ([n.d.]).

[34] Matthew Marge and Alexander I Rudnicky. 2013. Towards evaluating recovery strategies for situated grounding problems in human-robot dialogue. In 2013 IEEE RO-MAN. IEEE, 340-341.

[35] Marvin Minsky. 1968. Semantic information processing. (1968).

[36] Feng Nie, Jin-Ge Yao, Jinpeng Wang, Rong Pan, and Chin-Yew Lin. 2019. A simple recipe towards reducing hallucination in neural surface realisation. In Proceedings of the 57th Annual Meeting of the Association for Computational Linguistics. 26732679.

[37] Thomas Pellissier Tanon, Denny Vrandečić, Sebastian Schaffert, Thomas Steiner, and Lydia Pintscher. 2016. From Freebase to Wikidata: The great migration. In Proceedings of the 25th international conference on world wide web. 1419-1428.

[38] Jinfeng Rao, Kartikeya Upasani, Anusha Balakrishnan, Michael White, Anuj Kumar, and Rajen Subba. 2019. A Tree-to-Sequence Model for Neural NLG in Task-Oriented Dialog. In Proceedings of the 12th International Conference on Natural Language Generation. 95-100.

[39] Tanya Reinhart. 2016. Anaphora and semantic interpretation. Routledge.

[40] Elaine Rich. 1979. User modeling via stereotypes. Cognitive science 3, 4 (1979), 329-354.

[41] Samira Sheikhi and Jean-Marc Odobez. 2015. Combining dynamic head posegaze mapping with the robot conversational state for attention recognition in human-robot interactions. Pattern Recognition Letters 66 (2015), 81-90.

[42] Candace L Sidner, Christopher Lee, and Cory Kidd. 2005. Engagement during dialogues with robots. Proceedings of the Symposium on Conversational Informatics for Supporting Social Intelligence and Interaction: Situational and Environmental Information Enforcing Involvement in Conversation (2005), 27.

[43] Gabriel Skantze. 2008. Galatea: A discourse modeller supporting concept-level error handling in spoken dialogue systems. In Recent Trends in Discourse and Dialogue. Springer, 155-189.

[44] Gabriel Skantze and Anna Hjalmarsson. 2013. Towards incremental speech generation in conversational systems. Computer Speech \& Language 27, 1 (2013), 243-262.

[45] Gabriel Skantze, Anna Hjalmarsson, and Catharine Oertel. 2014. Turn-taking, feedback and joint attention in situated human-robot interaction. Speech Communication 65 (2014), 50-66.

[46] Daniel Szafir and Bilge Mutlu. 2012. Pay attention! Designing adaptive agents that monitor and improve user engagement. In Proceedings of the SIGCHI conference on human factors in computing systems. 11-20.

[47] Daniel Szafir and Bilge Mutlu. 2013. ARTFul: adaptive review technology for flipped learning. In Proceedings of the SIGCHI Conference on Human Factors in Computing Systems. 1001-1010.

[48] Denny Vrandečić and Markus Krötzsch. 2014. Wikidata: a free collaborative knowledgebase. Commun. ACM 57, 10 (2014), 78-85.

[49] Wolfgang Wahlster and Alfred Kobsa. 1989. User models in dialog systems. In User models in dialog systems. Springer, 4-34.

[50] Xue-feng Xi and Guodong Zhou. 2017. Building a Chinese discourse topic corpus with a micro-topic scheme based on theme-rheme theory. Big Data Analytics 2, 1 (2017), 9.

[51] Victor H Yngve. 1970. On getting a word in edgewise. In Chicago Linguistics Society, 6th Meeting, 1970. 567-578. 